\title{
Probability Forwarding Method in Mobile Wireless Sensor Networks
}

\author{
Haizheng $\mathrm{Yu}^{1}$ and Hong Bian ${ }^{2}$ \\ ${ }^{1}$ College of Mathematics and System Sciences, Xinjiang University, Urumqi, \\ 830046, China \\ ${ }^{2}$ School of Mathematical Sciences, Xinjiang Normal University, Urumqi, 830054, \\ China \\ \{haizheng_yu,bh1218\}@163.com
}

\begin{abstract}
Mobile wireless sensor networks without infrastructure are constructed including the mobile sink layer and the fixed sensor layer. The mobile sinks can communicate with the other mobile sinks. Every mobile sink receives data from the sensors and fuses to build a message. The message is copied, stored, and carried by mobile sinks and then it is transferred to the access point. In this paper, with multiple copies method of epidemic routing we present a probability forwarding scheme for message copies based on the threshold of nodes encountering each other. The method can decrease the message redundancy effectively, and control the waste of resource, bandwidth and energy arisen by a great deal of copies. At the same time, it guarantees the message transferred successfully. The theory analysis and the simulation experiment show that the delivery efficiency of our method is the same as one of epidemic routing, while the delay and the hop count do not increase and copies are fewer.
\end{abstract}

Keywords: Wireless sensor networks, Probability forwarding, Mobile sink

\section{Introduction}

Most wireless sensor networks (WSN) routing protocols are routing construction, maintenance and storage technology based on end-to-end routing, and the routing is dynamically maintained according to network topology. Some static routing cannot be used in WSN, because network topology is dynamically changed with mobility of sinks and nodes. Dynamic change reduces to unreliable communication links, and it is also very difficult to evaluate performance when locations of nodes are changed frequently.

However, in some scenes, the characteristic of nodes mobility can improve network performance. Liu [1] proposed that mobility improved network capacity and expanded network coverage. Wang [2] proposed that mobility prolonged the Time-to-Live of network. In mobile WSN, mobile nodes can share more works, and have more advantages comparing to static WSN [3]:

(1) In static WSN, the nodes near to the gateway sink always lose their energy firstly, which results to the overall network to "die". But in mobile WSN, because of the mobility, sensor nodes' energy consumption is more efficient.

(2) Mobile WSN is believed to have more channel capacity comparing with static WSN.

(3) Mobility helps for better quality of communication between sensor nodes. In a sparse or disconnected network, this property is especially helpful to maintaining efficient network connectivity.

(4) The probability of error increases with increasing number of hops that a data packet has to travel. If we reduce the number of hops, then the probability of error immediately reduces. 
In order to ensure messages forwarding successfully, the multiple copies methods are used as a method of message routing. However, multiple copies can create much message redundancy, which result in using up network resources. In the paper, we present a copy redundancy dropping scheme based on encountering count of contact probability among sinks. The scheme can ensure message forwarding successfully and decrease copies in the networks, thereby it can reduce the consumption of resource, buffer, bandwidth and energy.

\section{Mobile Wireless Sensor Networks}

The mobile WSN is two layer sensor networks building on the environment without infrastructure. The covering of mobile equipment, Smart phones, portables etc. act as moving converging sink in the upper layer. Besides of the basic moving function, the main function of these nodes is collecting data of sensor nodes, and handling the complicating computing, caching and transferring a lot of information. The lower layer is covering by the sensor nodes, which are arranged randomly, and have fixed positions. These nodes can immediately build the communication links with mobile equipment in the transmission range.

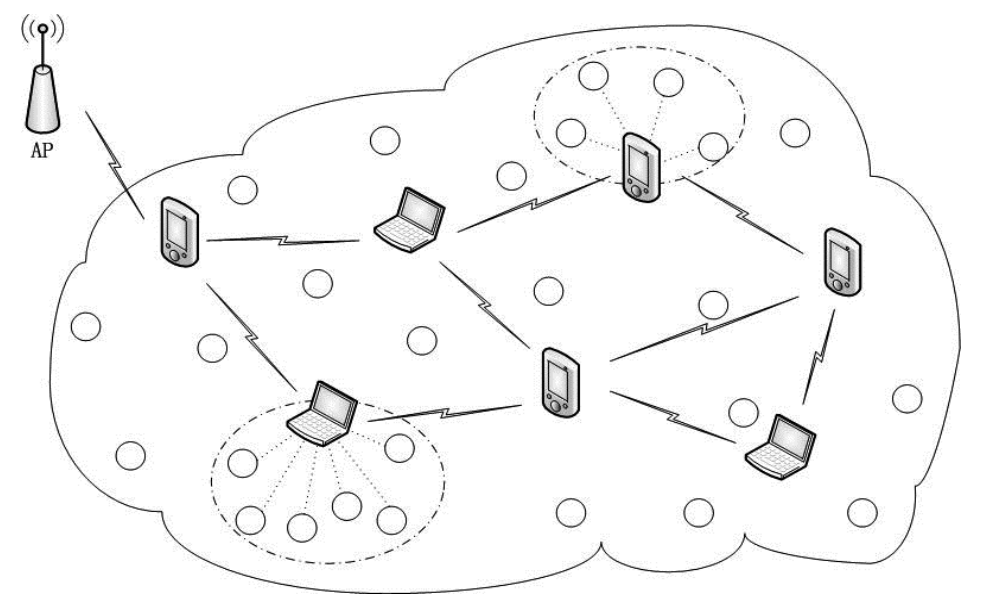

\section{Figure 1. Two-Tiered Mobile Wireless Sensor Network without Infrastructure}

The sensor nodes can communicate each other and build the ad hoc network. While considering the limit energy and function of the sensor nodes equipment, we require the sensor nodes only communicate with mobile equipment in order to maintain the long lifetime(See Figure1).

The mobile sink can obtain the data from the sensor nodes, fuse and storage, then through moving, hand it to others moving converging sink in the form of news, and deliver it successively. Finally arrive at access node AP. Aiming at the mobile sink sensor network, Gatzianas proposed a distribute algorithm for computer the maximum lifetime [4]. Because of frequently moving and the limit amount of the converging sink, which may lead to the intermittent connectivity of the network consisting of mobile equipment.

The technique of DTN (Delay Tolerant Networks) [5-7] has applied in wireless sensor networks, involving frequently intermittent environment. Wang propose a kind of frame of mobile sensor network based on DTN [8]: DFT-MSN (Delay/Fault-Tolerant Mobile Sensor Network), aiming at the moving nodes, loose connectivity, endurable mistaken, endurable delay and limit cache etc., he designs two transfer modes based on copy and news fault tolerant of self-adaption.

In this paper, aiming at the frequently moving and scattered distribution of mobile sink in the network, we reasonably resolve the news transmitting and redundancy eliminating 
between the mobile sinks, by making use of DTN frame technique, and adopting Epidemic routing [9].

\section{Probability Forwarding Scheme}

In this paper, we adopt the Random Waypoint moving model. All nodes satisfy the following conditions: (1)Evenly we select a coordinate $\mathrm{x}$ evenly in the range of network; (2)The node randomly selects a value of rate in a given $\left[\mathrm{v}_{\min }, \mathrm{v}_{\max }\right]$, move and point to $\mathrm{x}$; (3)When the node arrive at $\mathrm{x}$, suspend a time $\mathrm{t}, \mathrm{t} \in\left[\mathrm{T}_{\min }, \mathrm{T}_{\max }\right]$, then turn to step (1).

We make use of Epidemic routing to transmit news, and adopt the so-called "Storecarry-forward" mechanism, the source node $S$ first produce a new message $M, M$ is obtained by fusing the data, and communicates with $\mathrm{S}$. S is moving in the network, while S encounters a node A without copy $\mathrm{M}, \mathrm{S}$ copy $\mathrm{M}$ and transmit it to the node $\mathrm{A}$, A cache the copy of $M$, move and deliver the message $M$ to the encountering new node successively. As long as the node with the copy of M encounter the node without the copy of $\mathrm{M}$, the node copies $\mathrm{M}$ and deliver it to the node without the copy of $\mathrm{M}$ (which is similar to the spread of infectious disease), all mobile nodes deliver news in the same of infectious method, finally the node with the copy of $\mathrm{M}$ encounters the access node AP, and then deliver news to AP, which complete the process of deliver.

When the amount of communication is very low in the network, at the cost of increasing the space of cache, bandwidth and the energy of deliver, delay in deliver reaches minimum in the routing mechanism. However, message copies are infected and spread, which can increase network load, and lead to congestion of link and storage.

Definition 1. Encountering is a process in which one node enters the signal transmission range of another node and communicates each other until to leave.

Theorem 1. If we denote by $\alpha$ the encountering rate of two mobile nodes and E[V] the expectation of average relative rate of two nodes. Then we have

$$
\alpha \approx \frac{2 \omega r E[V]}{A}
$$

where $\mathrm{r}$ is the radius of delivering; $\mathrm{A}$ is the scope of the network; $\omega$ is a constant, but it has the different value in different mobile models $[10,11]$.

Theorem 2. If we denote by $\Gamma(t)$ the average encountering counter of two mobile nodes, then we have

$$
\Gamma(t)=\frac{1}{2} \alpha N(N-1) t
$$

where $\mathrm{N}$ is the number of nodes in the network[12].

Theorem 3. If $\mathrm{N}(\mathrm{t})$ is denoted by the average number function of infected nodes. Then we have

$$
N(t)=\frac{N}{1+(N-1) e^{-\alpha N t}}
$$

where $\mathrm{N}$ is the number of nodes in the network.

If $t$ equals to zero, the total number of copies about a message is one. In the initial state, the source node first generates a message. When $t$ tends to infinite, the total number of copies of a message is $N$, the message copies spread all over the nodes, which indicates that the message can be delivered to every node in the network.

There are two common methods to remove the copies of message in the network. One is TTL (Time-to-Live) method, which the copies are deleted by setting TTL of message. If TTL is little, the message could not be delivered successfully. If TTL is large, the 
message can be delivered to AP, but the copies of message are too large in the network, which results in congestion and other unfavorable factors.

Another is "vaccine" method, such as anti-packet[12]. The principle of the method is as following: if a message is delivered successfully, then the destination node releases an anti-packet for the message, while the node receiving the anti-packet immediately removes its copy of the message. Finally, the anti-packets spread in an epidemic manner.

In this paper, we do not set TTL of message and also do not create anti-packet for the message. With the increasing of the number of encountering counter, we can record the number of encountering between nodes carrying the same copy. The copy is deleted until a setting threshold is beyond. If each mobile node is healthy, our proposed method can not only guarantee the message delivery, also can reduce the copies of the message in the network, finally, it can completely eliminate the copies of the message.

\section{Experiment and Analysis}

In this paper we evaluate our probability forwarding method in the convergence layer. In the layer, we receive the time of delivering successfully when the copies of message are dropped and the time of removing completely. By the simulation experiments: $500 \mathrm{~m} \times 500 \mathrm{~m}, 20$ mobile sinks, speed: $v \in[1 \mathrm{~m} / \mathrm{s}, 8 \mathrm{~m} / \mathrm{s}]$, signal range radius: $r=10 \mathrm{~m}, 2.4 \mathrm{GHz}$ communication channel, interval time: $t \in[0 s, 8 s]$, and independent Random Waypoint mobility model. An access point is installed in the boundaries of network (see Figure 1). Simulation tool is OMNet++4.4 [13].

We compare with the effects of two cases of Epidemic routing to deliver successfully and delay. One performs removal method, and the other does not. As shown in Figure 2, by setting the appropriate eliminating threshold will make all of the mobile sinks deliver message to AP successfully.

When the value of the threshold is three, we find that the delay is the same as that of no setting threshold. But the copies of message are significantly reduced in the network.

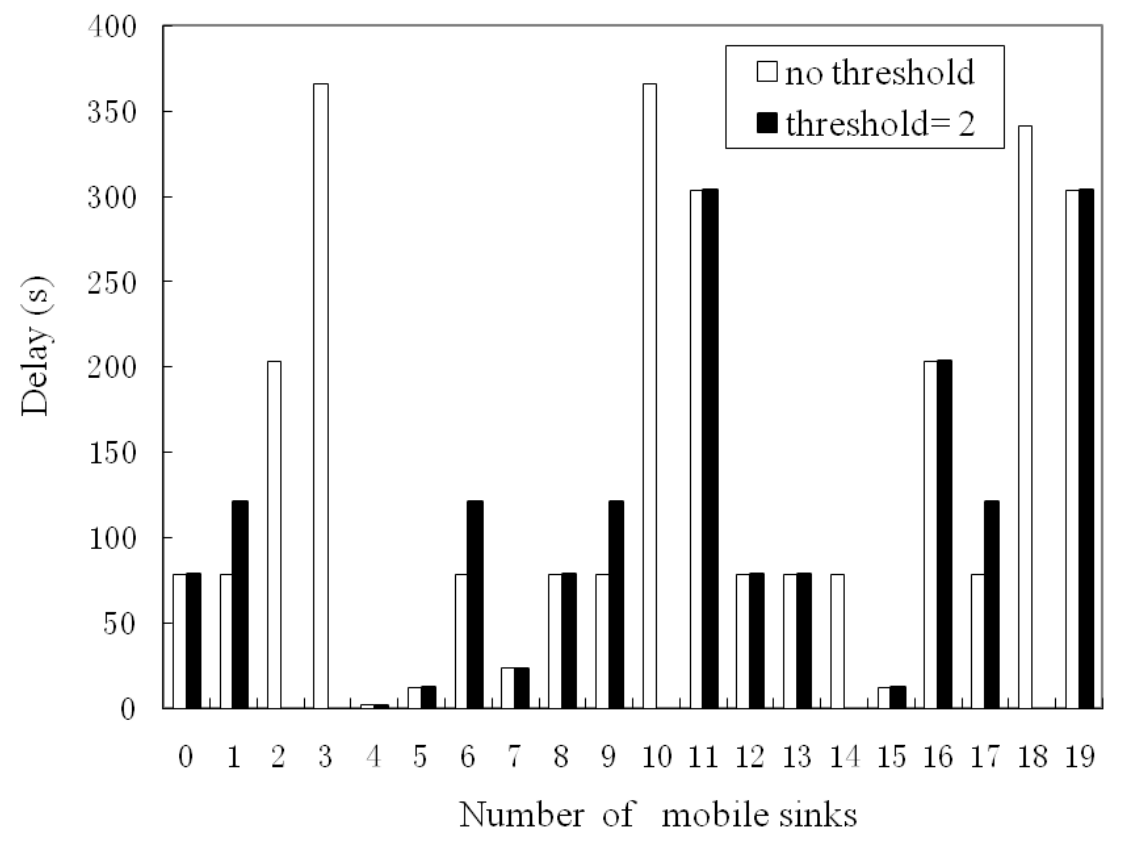

Figure 2. Delay of the Message Copies Reaching the AP

See Figure 2, the message delay whose threshold is 2 is much longer than that of no threshold, when the moving sinks are 1, 6, 9, 17, the message can deliver successfully. 
While the moving sinks are $2,3,10,14,18$, the message can't deliver to AP, because the threshold of message deleting is more lower, which result that the message copies have been removed completely before it arrive at AP; at the same time, Hop which part of messages are deliver to AP successfully is more than that of no threshold.

Table 1. Hop Count with the Different Thresholds

\begin{tabular}{|c|c|c|c|}
\hline $\begin{array}{c}\text { Source } \\
\text { node index }\end{array}$ & $\begin{array}{l}\text { Hop counts } \\
\text { (no } \\
\text { threshold) }\end{array}$ & $\begin{array}{r}\text { Hop counts } \\
\text { ( threshold }=2 \text { ) }\end{array}$ & $\begin{array}{r}\text { Hop counts } \\
\text { ( threshold }=3 \text { ) }\end{array}$ \\
\hline $\mathbf{0}$ & 2 & 2 & 2 \\
\hline 1 & 2 & 3 & 2 \\
\hline 2 & 4 & $\infty$ & 4 \\
\hline 3 & 4 & $\infty$ & 4 \\
\hline 4 & 1 & 1 & 1 \\
\hline 5 & 2 & 2 & 2 \\
\hline 6 & 3 & 4 & 3 \\
\hline 7 & 1 & 1 & 1 \\
\hline 8 & 2 & 2 & 2 \\
\hline 9 & 2 & 3 & 2 \\
\hline 10 & 3 & $\infty$ & 3 \\
\hline 11 & 2 & 2 & 2 \\
\hline 12 & 3 & 3 & 3 \\
\hline 13 & 4 & 4 & 4 \\
\hline 14 & 3 & $\infty$ & 3 \\
\hline 15 & 1 & 1 & 1 \\
\hline 16 & 2 & 2 & 2 \\
\hline 17 & 1 & 3 & 1 \\
\hline 18 & 5 & $\infty$ & 5 \\
\hline 19 & 3 & 3 & 3 \\
\hline
\end{tabular}

Setting proper threshold of removal can ensure all mobile sink nodes transfer message to AP successfully. As shown in Table 1, in the following three cases: there is no threshold, the threshold is two, and the threshold is three, we compare with hop counts in three cases. When the threshold is 3 , we find that hop counts are same with no threshold.

A mobile sink receives data collected by sensors, and fuses to create message to deliver to AP. In simulation, a sink is selected randomly, and send a message to AP with no removal threshold (Epidemic routing) and carrying threshold (values are 2, 3, 4, 5, 6).

We compare the copies number among different threshold in Figure 3, and conclude that the number of copies is less when the threshold is lower, at the same time, the copies of message are deleted completely more quickly.

By Theorem 2, the number of encountering $\Gamma$ and time $t$ are linear relationship approximately, and we regard the number of encountering as $\mathrm{x}$ axis to replace by time $\mathrm{t}$ in order to data statistics. 


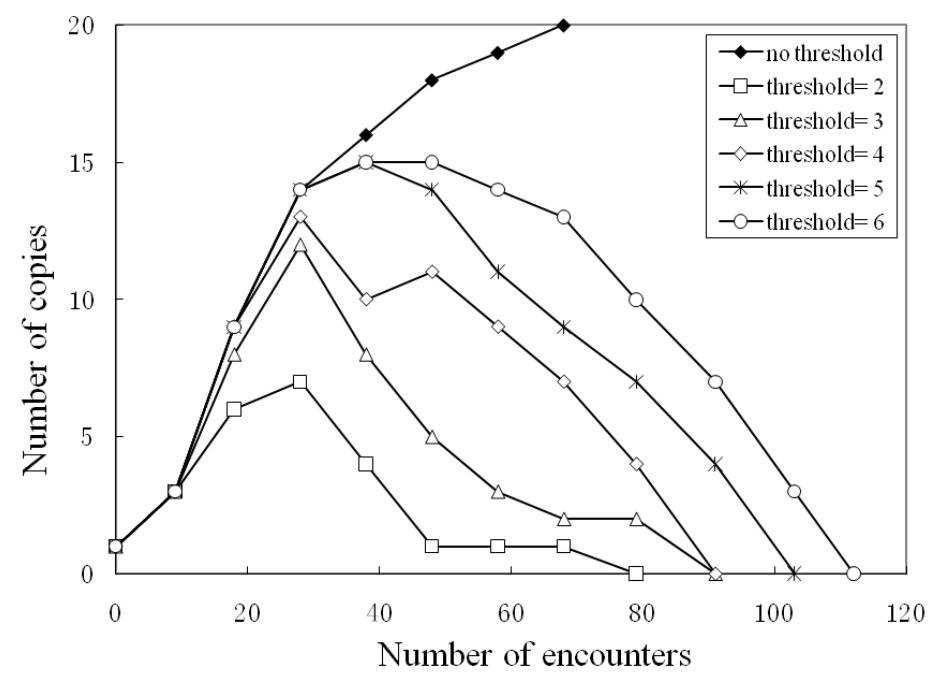

Figure 3. The Copies of Message with the Different Thresholds

\section{Conclusion}

Two layers in mobile wireless sensor network are built on the environment without infrastructure. The moving converging sinks are in the upper layer and sensor nodes are in the lower layer, which are arranged randomly with fixed positions. Mobile sinks get data from sensors and fuse them into a message. The message is forwarded with probability among mobile sinks with DTN technology, finally the message is delivered to access point.

Using Epidemic routing can ensure message delivering successfully to AP, but creates a lot of copies of the message to increase network load. With probability encountering of mobile sinks, we can record the number of encountering, by which the redundant copies are dropped according to the setting threshold. By selecting proper threshold, we can ensure message delivering successfully and decrease the copies of message in the network.

\section{Acknowledgment}

The work is supported by the National Natural Science Foundation of China (11361062, 61262089, 61262087), Key Program of Xinjiang Higher Education (XJEDU2012I28, XJEDU2013I04), Research Fund for the Doctoral Program of Xinjiang University (BS110103), Key Program of Xinjiang Natural Science Foundation (2013211A021).

\section{References}

[1] B. Liu, P. Brass, O. Dousse and P. Nain, D. Towsley, "Mobility Improves Coverage of Sensor Networks", Proceedings of the 6th ACM international symposium on Mobile ad hoc networking and computing (MobiHoc), (2005) Illinois, pp. 300-308,

[2] W. Wang, V. Srinivasan and K.C. Chua, "Using Mobile Relays to Prolong the Lifetime of Wireless Sensor Networks", Proceedings of the 11th annual international conference on Mobile computing and networking (MobiCom), (2005) Cologne. pp. 270 -283.

[3] S. A. Munir, B. Ren, W. W. Jiao, B. Wang, D. L. Xie and J. Ma, " Mobile wireless sensor network: Architecture and enabling technologies for ubiquitous computing", Proceedings of 21st International Conference on Advanced Networking and Applications Workshops/Symposia (AINAW), vol. 2, (2007) Canada, pp. 113-120,

[4] M. Gatzianas and L. Georgiadis, "A Distributed Algorithm for Maximum Lifetime Routing in Sensor Networks with Mobile Sink", IEEE Transactions on Wireless Communications, vol. 7, no. 3, (2008), pp. 984-994. 
[5] K. Fall, "A Delay-tolerant network architecture for challenged internets", Proceedings of ACM SIGCOMM, (2003) Germany, pp. 27-34.

[6] S. Burleigh, A. Hooke , L. Torgersonn, K. Fall, V. Cerf, B. Durst, K. Scott and H. Weiss, "Delay Tolerant Networking - An Approach to Interplanetary Internet", IEEE Communications Magazine, vol. 41, no. 6, (2003), pp. 128-136.

[7] A. Voyiatzis, "A Survey of Delay- and Disruption-Tolerant Networking Applications", Journal of Internet Engineering, vol. 5, no. 1, (2012), pp. 331-344,

[8] Y. Wang and H. Y. Wu, "Delay/Fault-Tolerant Mobile Sensor Network (DFT-MSN): A New Paradigm for Pervasive Information Gathering", IEEE Transactions on Mobile Computing, vol. 6, no. 9, (2007), pp. 1021-1034.

[9] A. Vahdat and D. Becker, "Epidemic routing for partially connected ad hoc networks", Duke University, Technical Report CS-200006,( 2000).

[10] R. Groenevelt, P. Nain and G. Koole, "The message delay in mobile ad hoc networks", Elsevier Journal of Performance Evaluation, vol. 62, (2005), pp. 210-228.

[11] R. Groenevelt, "Stochastic Models for Ad hoc Networks", PhD thesis, France: INRIA Sophia Antipolis, (2005)

[12] H. Z. YU, J. F. Ma and H. Bian, "Reasonable Routing in Delay /Disruption Tolerant Networks ", Frontiers of Computer Science in China, vol. 5, no. 3, (2011), pp. 327-334.

[13] OMNeT++4.4, http://www.omnetpp.org, (2013).

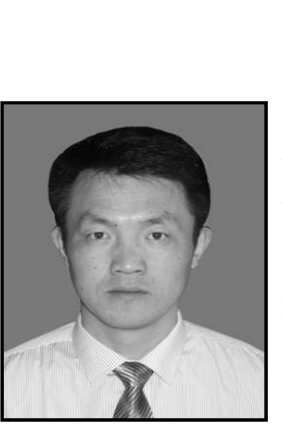

\section{Authors}

Haizheng Yu, he is an associate professor from Xinjiang University, P. R. China. He received his Ph. D. degree from Xidian University, Xi'an, P. R. China in 2011. He is currently a visiting scholar of Wuhan University. His research interests include wireless networks, mobile networks and network security.

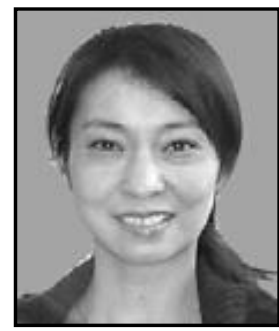

Hong Bian. She is a professor from the school of Mathematical Sciences, Xinjiang Normal University, P.R. China. She received her $\mathrm{Ph}$. D. degree in apply mathematics from Xiamen University in 2008. Her research interests include graph theory and network optimization. 
International Journal of Future Generation Communication and Networking Vol. 8, No. 6 (2015) 